\title{
A GENERALIZATION OF THE THREE-DIMENSIONAL MACPHERSON-SROLOVITZ FORMULA*
}

\author{
THINH $\mathrm{LE}^{\dagger}$ AND QIANG DU
}

\begin{abstract}
The MacPherson-Srolovitz formula has been recently established as a generalization of the two dimensional von Neumann relation for microstructure coarsening. In this paper, we present an extension of the MacPherson-Srolovitz formula under more general junction conditions.
\end{abstract}

Key words. Microstructure evolution, Three dimensional grain growth, Grain volume kinetics, Von Neumann-Mullins formula, Herring condition, Mean width.

AMS subject classifications. 74N15, 74N20, 51P05.

\section{Introduction}

The growth of grains (in metals, ceramics, etc) and bubbles (in froths, foams) has been a topic of active research for many years. We hereby refer the grain cells (or bubbles) as domains. Relatively speaking, the two-dimensional growth process is much better understood. A good example is the von Neumann-Mullins formula $[11,12]$ which states that

$$
\frac{d A}{d t}=-2 \pi M \gamma\left(1-\frac{1}{6} n\right)
$$

Here $\frac{d A}{d t}$ is the rate of change of the area of a $2 \mathrm{D}$ domain, $n$ is the number of triple junctions around the domain, $M$ is a mobility constant of the domain wall (interface, boundary, with co-dimension 1) with $\gamma$ being the surface tension.

In [9], MacPherson and Srolovitz derived some generalizations of (1.1) to any space dimension. Their formula for the three-dimensional case, in particular, is as follows

$$
\frac{d V}{d t}=-2 \pi M \gamma\left(\mathcal{L}(\mathbf{D})-\frac{1}{6} \sum_{i=1}^{n} e_{i}(\mathbf{D})\right)
$$

Here, $\frac{d V}{d t}$ is the rate of change of the volume $V$ of a closed three-dimensional domain D having piecewise smooth boundary and with any two of its faces meeting along a triple line (of the grain system) at a fixed dihedral angle of $2 \pi / 3, \mathcal{L}(\mathbf{D})$ is the mean width of $\mathbf{D}, n$ is the number of triple lines (edges) of $\mathbf{D}, e_{i}(\mathbf{D})$ is the length of $i$-th triple line and the summation is over all $n$ triple lines.

For an arbitrary domain $\mathbf{D}$, its mean width can be defined as follows: for each straight line $\ell$ through the origin of some coordinate system in the three dimensional space, we define the Euler width of $\mathbf{D}$ in the direction $\ell$ as $E_{\ell}(\mathbf{D})=\int_{\ell} \chi\left(\ell_{p}^{\perp} \cap \mathbf{D}\right) d p$,

${ }^{*}$ Received: November 20, 2008; accepted (in revised version): February 17, 2009. Communicated by Jack Xin.

This research is supported in part by NSF DMS-0712744.

${ }^{\dagger}$ Department of Mathematics, Pennsylvania State University, University Park, PA 16802 (le@math.psu.edu).

${ }^{\ddagger}$ Department of Mathematics and Department of Materials Science and Engineering, Pennsylvania State University, PA 16802 (qdu@math.psu.edu). http://www.math.psu.edu 
the integral is over all points $p$ along $\ell, \chi\left(\ell_{p}^{\perp} \cap \mathbf{D}\right)$ is the Euler characteristic of the intersection of the domain $\mathbf{D}$ with the plane $\ell_{p}^{\perp}$ perpendicular to the line $\ell$ at the point $p$. The mean width of $\mathbf{D}, \mathcal{L}(\mathbf{D})$, if it exists, is twice the Euler width averaged over all lines $\ell$ through the origin. For more details about the mean width of a domain we refer to $[6,9,14]$.

When $\mathbf{D}$ is a closed convex three dimensional domain with a piece-wise smooth boundary the quantity $(1 / 2) \mathcal{L}(\mathbf{D})$ equals to the so-called mean caliper diameter (for instance see [14]) which is the mean distance between parallel support planes of $\mathbf{D}$. In this case if the convex domain $\mathbf{D}$ also satisfies the same conditions specified in the above for the formula (1.2), then the following formula has been given in [3]:

$$
\frac{d V}{d t}=-2 \pi \bar{M}\left(\bar{D}-\frac{1}{12} L\right)
$$

where $\bar{D}$ is the caliper diameter of the domain, $\bar{M}$ is mentioned as an empirical proportionality constant which we interpret as $2 M \gamma$ and $\mathrm{L}$ is stated as the edge length which we interpret as the total length of all edges of the domain. Thus, Cahn's formula (1.3) can be considered to be the same as the formula (1.2) for a convex domain.

Cahn's formula (1.3) and the more general MacPherson-Srolovitz formula (1.2) provide insight to the important mathematical question on the grain growth in an ideal setting. As noted in [8], much work remains to be investigated due to the simplifying assumptions used by Neumann-Mullins and MacPherson-Srolovitz. For instance, factors which are ignored include the inhomogeneities and anisotropy in energy, the nonuniform mobilities and the drag induced by the junctions. It is interesting to study if a generalized MacPherson-Srolovitz formula can be obtained by relaxing the underlying assumptions. In this work, the particular assumption we focus on is the triple junction condition (a special case of the Herring condition) which is further elaborated in section 2 of this paper. We show, through simple calculations based on $[9]$, that the following extension can be obtained

$$
\frac{d V}{d t}=-2 \pi M \gamma\left(\mathcal{L}(\mathbf{D})-\frac{1}{2 \pi} \sum_{i=1}^{n} \int_{e_{i}(\mathbf{D})} \alpha_{i} d l\right)
$$

under more general conditions that allow arbitrary $k$-junction lines (for $k \geq 3$ ) with a set of corresponding dihedral exterior angles $\left\{\alpha_{i}\right\}$. With the help of some preliminary discussions given in section 3, the extended formula (1.4) is derived in section 4 . The derivation utilizes a simple but key observation on the mean width computation for three dimensional domains. We also discuss some consequences of the generalized formula on the grain growth process at the end of the paper.

\section{Junctions, Herring condition, and mean width}

We note that in deriving the Neumann-Mullins and MacPherson-Srolovitz formulae, a boundary condition on the junctions (points in two dimensions and curves or lines in the three dimensions) is used. It is assumed that only three (co-dimension 1) domain walls meet along a (co-dimension 2) junction (which is the most generic case) and they meet at the (turning) angle of $\pi / 3$. Equivalently, this is also often phrased as the three domain walls meeting at $120^{\circ}$. Such a junction condition can be seen as a natural boundary condition for the interface motion (variation), which is implied by the Herring condition (for the ideal isotropic grain growth) at the triple junction. The Herring condition in three dimensions assumes the mechanical balance of forces in the 
plane perpendicular to a junction, which means that a junction does not affect grain boundary motion through an explicit driving force. However, the spatial orientation of each junction also depends on interface properties and each interface contributes some torque acting towards the change of the junction direction. It has been shown that each junction does possess its own mobility as an additional kinetic parameter and thus it does influence the kinematics of grain microstructure evolution. For a triple junction, if its mobility is relatively low, the angle between two interfaces may tend to 0 , in contrast with the Herring Condition, and the influence of triple junctions results in a drag or reduced rate of microstructure evolution during grain growth. We refer to [5] and [10] for more details.

The main contribution of this work is to provide an extension of (1.2) when the triple junction condition is omitted. We focus on the three dimensional case as this is most relevant to the study of grain growth and microstructure coarsening in a practical sense. In deriving the formula, some geometric consideration similar to that in [9] needs to be taken into account. In particular, we make a simple but key observation that the mean width of a polyhedron $\mathbf{D}$ with flat faces and $n$ edges satisfies

$$
\mathcal{L}(\mathbf{D})=\frac{1}{2 \pi} \sum_{i=1}^{n} e_{i} \alpha_{i}
$$

where $e_{i}$ is the length of edge $i$ and $\alpha_{i}$ is the exterior (turning) angle of $\mathbf{D}$ at edge $i$. The above formula holds for both convex and non-convex polyhedra and is proved later. If $\mathbf{D}$ is concave along an edge $i$, then the turning angle $\alpha_{i}$ is negative. In fact, the dihedral exterior angle can be defined as the difference of $\pi$ with the dihedral interior angle, so that any exterior angle belongs to the interval $(-\pi, \pi)$.

On the other hand, since all faces of $\mathbf{D}$ are flat, we have

$$
\int_{\partial \mathbf{D}} \mathcal{K} d s=0=2 \pi\left(\mathcal{L}(\mathbf{D})-\frac{1}{2 \pi} \sum_{i=1}^{n} e_{i} \alpha_{i}\right)
$$

Here, we use $\mathcal{K}$ to denote the mean curvature (the average of the two principal curvatures) of a surface with $d s$ being the area element.

Drawing the similarity with the terms used in the MacPherson-Srolovitz formula, this suggests that in general, for any given domain $\mathbf{D}$ with a set of edges $\left\{e_{i}(\mathbf{D})\right\}_{i=1}^{n}$ and corresponding exterior dihedral angles $\left\{\alpha_{i}\right\}_{i=1}^{n}$, the following identity could be true:

$$
\int_{\partial \mathbf{D}} \mathcal{K} d s=2 \pi\left(\mathcal{L}(\mathbf{D})-\frac{1}{2 \pi} \sum_{i=1}^{n} \int_{e_{i}(\mathbf{D})} \alpha_{i} d l\right)
$$

When all exterior angles are $\pi / 3$, this reduces to the result of MacPherson-Srolovitz [9]. The more general case is proved later in the paper.

\section{Preliminary}

We now present some necessary technical background to our discussion, mostly following the work of [9]. We begin with some basic assumptions. Let $\mathbf{D}$ be a closed three-dimensional domain with a piece-wise smooth boundary $\partial \mathbf{D}$. A smooth component of $\partial \mathbf{D}$ is referred as a wall and the number of walls remains finite. Each (co-dimension 2) edge of the domain is the intersection of two walls, but note that the edge we consider here does not have to be limited to the triple lines. At each point on 


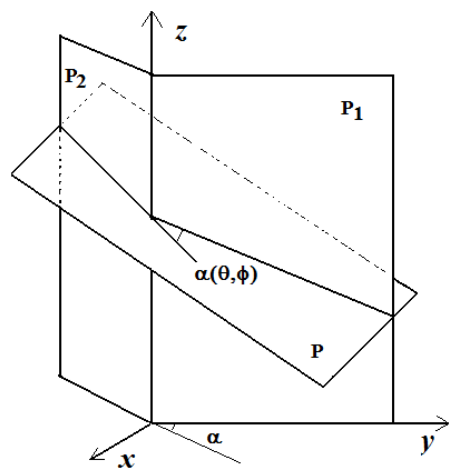

FIG. 3.1. The dihedral exterior angle $\alpha$ of $\mathbf{D}$ and the exterior angle $\alpha(\theta, \phi)$ in the plane $P$. The corresponding interior angles are $\beta=\pi-\alpha$ and $\beta(\theta, \phi)=\pi-\alpha(\theta, \phi)$

an edge, there are two half tangent planes corresponding to two walls of the domain so that the dihedral interior angle $\beta$ is defined uniquely in the plane perpendicular to the edge at such a point. The dihedral exterior angle can then be defined as $\alpha=\pi-\beta$.

Given a coordinate system in three dimensional space, we can consider the corresponding spherical coordinates $(r, \theta, \phi)$, where $r$ runs from $-\infty$ to $\infty, \theta$ runs from 0 to $2 \pi$ and $\phi$ runs from 0 to $\pi / 2$. Each pair $(\theta, \phi)$ gives a direction in the three dimensional space and we denote $P=P(\theta, \phi)$ a plane perpendicular to that direction (note that we only need the direction of this plane since we will only work with angles). Moreover given a pair of half planes $\left(P_{1}, P_{2}\right)$ with the dihedral angle $\beta$, for any plane $P=P(\theta, \phi)$, we have a corresponding exterior angle $\alpha(\theta, \phi)$ and interior angle $\beta(\theta, \phi)=\pi-\alpha(\theta, \phi)$ (see figure 3.1 for an illustration). We know that the rate of change of the volume of a domain is given by a physical constant times the integral of the mean curvature $\mathcal{K}$ over the walls of the domain. The main point in the proof of the MacPherson-Srolovitz formula is following: for a planar domain $D$ in two dimensions which may have several disconnected components, each of which may have holes we have

$$
\int_{\partial D} \kappa d l=2 \pi \chi(D)-\sum_{j} \alpha_{j}[\partial D]
$$

Here $D$ is bounded by a curve $\partial D$ which consists of smooth sections meeting at points where the exterior angles are $\alpha_{j}[\partial D], \kappa$ is the curvature of $\partial D, \chi(D)$ is the Euler characteristic of $\mathrm{D}, d l$ is the length element of $\partial D$.

Now, in three dimensional space, we consider the set of all planes $\{\mathrm{P}\}$, which is again a three dimensional space with a natural volume element $d \Omega$ (independent of the coordinate system chosen). Integrating, over the set $\{\mathrm{P}\}$, both sides of the formula (3.1) restricted to the intersection of $P$ and the three dimensional domain $\mathbf{D}$ and multiplying both sides by 2 , we obtain

$$
2 \int_{\{P\}}\left(\int_{\partial(\mathbf{D} \cap P)} \kappa d l\right) d \Omega=2 \cdot 2 \pi \int_{\{P\}} \chi(\mathbf{D} \cap P) d \Omega-2 \int_{\{P\}} \sum_{j} \alpha_{j}[\partial(\mathbf{D} \cap P)] d \Omega .
$$

Then, the following relations were established [9]:

$$
2 \int_{\{P\}}\left(\int_{\partial(\mathbf{D} \cap P)} \kappa d l\right) d \Omega=\int_{\partial \mathbf{D}} \mathcal{K} d s
$$




$$
2 \cdot 2 \pi \int_{\{P\}} \chi(\mathbf{D} \cap P) d \Omega=2 \pi \mathcal{L}(\mathbf{D})
$$

and

$$
2 \int_{\{P\}} \sum_{j} \alpha_{j}[\partial(\mathbf{D} \cap P)] d \Omega=\sum_{i=1}^{n} \int_{e_{i}(\mathbf{D})} W_{i} d l
$$

with

$$
W_{i}=\frac{1}{\pi} \int_{\theta=0}^{2 \pi} \int_{\phi=0}^{\pi / 2} \alpha_{i}(\theta, \phi) \sin \phi \cos \phi d \theta d \phi
$$

being the average of the exterior angles $\alpha_{i}(\theta, \phi)$ at a point on the edge $e_{i}(\mathbf{D})$.

In [9], it was further proven that $W_{i} \equiv \pi / 3$ if the triple junction condition mentioned above is satisfied. Here we claim that this quantity can be explicitly computed in general.

For the lemma below, we assume that without loss of generality the pair of half planes $\left(P_{1}, P_{2}\right)$ has the $z$-axis as the common edge and we fix a direction of rotation to be along the $z$-axis. In this way, if the half plane $P_{1}$ is chosen as $\{O y z: y \geq 0\}$, then for any $\beta \in[0,2 \pi)$ there is a unique half plane $P_{2}$ such that the dihedral angle between $P_{1}$ and $P_{2}$ is $\beta$.

LEMMA 3.1. For each pair $\left(P_{1}, P_{2}\right)$ with the dihedral angle $\beta$, consider the average angle

$$
\hat{W}=\frac{1}{\pi} \int_{\theta=0}^{2 \pi} \int_{\phi=0}^{\pi / 2} \beta(\theta, \phi) \sin \phi \cos \phi d \theta d \phi .
$$

Then $\hat{W}$ is the identity function of the dihedral angle $\beta$ on $[0,2 \pi)$.

Proof. First we claim that $\hat{W}$ is invariant under any rotation around the $z$-axis (which is the chosen direction specified above). In fact if we rotate the pair $\left(P_{1}, P_{2}\right)$ with an angle $\delta$, then $\beta(\theta, \phi) \mapsto \beta(\delta+\theta, \phi)$. This is because when we rotate around the $z$-axis, the $z$-direction is fixed and, at the same time, a rotation with angle $\delta$ is done in the $O x y$ plane (or any plane which is parallel to $O x y$ ). So $\hat{W}$ is indeed a function of the variable $\beta$.

Since $\hat{W}$ is invariant under any rotation around the z-axis, we see that $\hat{W}\left(\beta_{1}+\right.$ $\left.\beta_{2}\right)=\hat{W}\left(\beta_{1}\right)+\hat{W}\left(\beta_{2}\right)$ for any $\beta_{1}, \beta_{2} \in[0,2 \pi)$ satisfying $\beta_{1}+\beta_{2} \in[0,2 \pi)$. In fact, let us choose $P_{1}$ as above, then there is a unique $P_{2}$ such that the dihedral angle between $P_{1}$ and $P_{2}$ is $\beta_{1}$ and a unique $P_{3}$ such that the dihedral angle between $P_{2}$ and $P_{3}$ is $\beta_{2}$. Thus $\beta_{1}+\beta_{2}$ corresponds to the pair $\left(P_{1}, P_{3}\right)$ and it is clear that the angle $\beta(\theta, \phi)$ corresponding to this pair is the sum of the ones corresponding to the pairs $\left(P_{1}, P_{2}\right)$ and $\left(P_{2}, P_{3}\right)$.

Moreover, it is obvious that $\hat{W}$ is an increasing function of its argument, and $\hat{W}$ is continuous. Indeed, a direct calculation leads to

$$
\cos (\beta(\theta, \phi))=\frac{\cos \beta+\cot ^{2} \phi \sin \theta \sin (\beta+\theta)}{\sqrt{\left(1+\cot ^{2} \phi \sin ^{2} \theta\right)\left(1+\cot ^{2} \phi \sin ^{2}(\beta+\theta)\right)}} .
$$

It is also clear that $\hat{W}(\pi)=\pi$.

These above conditions force $\hat{W}$ to be the identity function on $[0,2 \pi)$ and the lemma is proved. 
Next, as defined above, for an edge $e_{i}(\mathbf{D})$ of the domain $\mathbf{D}$ we consider

$$
W_{i}=\frac{1}{\pi} \int_{\theta=0}^{2 \pi} \int_{\phi=0}^{\pi / 2} \alpha_{i}(\theta, \phi) \sin \phi \cos \phi d \theta d \phi
$$

at a point on that edge with the exterior angle $\alpha_{i}$. Then we have

Lemma 3.2. For a point on the edge $e_{i}(\mathbf{D})$ of the domain $\mathbf{D}$, the quantity $W_{i}$ is exactly the value of the exterior angle $\alpha_{i}$.

Proof. Here, without loss of generality, we choose a spherical coordinate system so that the $z$-axis is tangent to the curve $e_{i}(\mathbf{D})$ at the point, and one of the half tangent planes of $\mathbf{D}, P_{1}$, is the half plane $\{O y z \mid y \geq 0\}$ (two half tangent planes of the domain $\mathbf{D}$ give the dihedral interior angle $\left(\pi-\alpha_{i}\right)$ of the domain).

From now on we suppress the index $i$ for simplicity. Let $\beta=\pi-\alpha$, or more precisely, let $\beta(\theta, \phi)=\pi-\alpha(\theta, \phi)$ denote the interior angle. We have

$$
W=\pi-\frac{1}{\pi} \int_{\theta=0}^{2 \pi} \int_{\phi=0}^{\pi / 2} \beta(\theta, \phi) d \theta \sin \phi \cos \phi d \phi=\pi-\hat{W}(\beta) .
$$

By Lemma 3.1 on $[0,2 \pi)$, the function

$$
\hat{W}(\beta)=\frac{1}{\pi} \int_{\theta=0}^{2 \pi} \int_{\phi=0}^{\pi / 2} \beta(\theta, \phi) d \theta \sin \phi \cos \phi d \phi
$$

is an identity map. Thus, $W$ is exactly the dihedral exterior angle $\alpha$.

\section{Main results}

We now state the main results of this paper. The first one is on the characterization of the mean width presented in section 2 .

THEOREM 4.1. Under the above assumptions on the domain $\mathbf{D}$ with edges $\left\{e_{i}(\mathbf{D})\right\}_{i=1}^{n}$ and the set of corresponding dihedral exterior angles $\left\{\alpha_{i}\right\}_{i=1}^{n}$, we have

$$
\int_{\partial \mathbf{D}} \mathcal{K} d s+\sum_{i=1}^{n} \int_{e_{i}(\mathbf{D})} \alpha_{i} d l=2 \pi \mathcal{L}(\mathbf{D})
$$

Proof. Based on the work by MacPherson and Srolovitz in [9], we know that, as briefly outlined in the discussion before Lemma 3.1,

$$
\int_{\partial \mathbf{D}} \mathcal{K} d s+\sum_{i=1}^{n} \int_{e_{i}(\mathbf{D})} W_{i} d l=2 \pi \mathcal{L}(\mathbf{D}) .
$$

By Lemma 3.2, for any point on the edge $e_{i}(\mathbf{D})$ we have

$$
W_{i}=\frac{1}{\pi} \int_{\theta=0}^{2 \pi} \int_{\phi=0}^{\pi / 2} \alpha_{i}(\theta, \phi) d \theta \sin \phi \cos \phi d \phi
$$

is exactly the value of the exterior angle $\alpha_{i}$. Replacing $W_{i}$ by $\alpha_{i}$, we immediately obtain (4.1).

In deriving the Neumann-Mullins and MacPherson-Srolovitz formulas, it has been assumed that all edges are triple lines and a force balance is maintained along edges so that three domain walls meet along an edge with turning angles all having the 
same value $\pi / 3$. With the help of the formula (4.1) in the above theorem, we can derive a more general formula by omitting these restrictions so that an edge can be any $k$-junction line for some $k \geq 3$, and furthermore, there is no need to enforce the force balance at these edges so that the exterior angles may vary between different values.

Theorem 4.2. (The extension to the three dimensional MacPherson-Srolovitz formula for more general junction conditions).

Suppose that we have a system of evolving grains governed by the Mullins equation such that all grains have the same interfacial mobility constant $M$ and interface surface tension $\gamma$. Then the rate of change of the volume $V$ of an individual grain $\mathbf{D}$ with the set of edges $\left\{e_{i}(\mathbf{D})\right\}_{i=1}^{n}$ (edges are $k$-junction lines for some $k \geq 3$ ) and the set of corresponding dihedral exterior angles $\left\{\alpha_{i}\right\}_{i=1}^{n}$ is given by:

$$
\frac{d V}{d t}=-2 \pi M \gamma\left(\mathcal{L}(\mathbf{D})-\frac{1}{2 \pi} \sum_{i=1}^{n} \int_{e_{i}(\mathbf{D})} \alpha_{i} d l\right) .
$$

Proof. The Mullins equation states that the normal velocity of the domain walls satisfies

$$
v_{n}=M \gamma \mathcal{K},
$$

with $\mathcal{K}$ being the surface mean curvature. We thus have

$$
\frac{d V}{d t}=-\int_{\partial \mathbf{D}} v_{n} d s=-M \gamma \int_{\partial \mathbf{D}} \mathcal{K} d s .
$$

Using the identity (4.1), the formula (4.2) then readily follows from the above.

Consequently, the three-dimensional MacPherson-Srolovitz formula is a special case of the formula (4.2).

Corollary 4.3. With the same assumptions as in the above theorem, if we assume

additionally that along any $k$-junction line $e_{i}^{(k)}(\mathbf{D})$ the exterior angle is $\left(\pi-\frac{2 \pi}{k}\right)$ (thus the force balance is maintained along all edges of grains), then we obtain

$$
\frac{d V}{d t}=-2 \pi M \gamma\left(\mathcal{L}(\mathbf{D})-\frac{1}{2} \sum_{k \geq 3} \sum_{i=1}^{n(k)}\left(1-\frac{2}{k}\right) e_{i}^{(k)}(\mathbf{D})\right) .
$$

Here the first sum is taken over all possible $k$ such that a $k$-junction line is present, $n(k)$ is the number of $k$-junction lines and $\sum n(k)=n$ (the total number of edges of the domain). In particular, if all junctions are triple-junctions, we have the MacPhersonSrolovitz formula

$$
\frac{d V}{d t}=-2 \pi M \gamma\left(\mathcal{L}(\mathbf{D})-\frac{1}{6} \sum_{i=1}^{n} e_{i}^{(3)}(\mathbf{D})\right)
$$

\section{Discussions}

The formula (4.2), which is the same as (1.4), is one of the possible generalizations of the work in [9] which relaxes the Herring condition. The latter is often a 
key ingredient to ensure the energy dissipation in the grain growth process. Indeed, consider an evolving family of interfacial networks

$$
\left\{S:(x, t) \mapsto u(x, t) \in \mathbb{R}^{3}, x \in \Omega, t>0\right\}
$$

where $\Omega$ is a domain in $\mathbb{R}^{2}$.

Any surface $\mathrm{S}$ in this network has the normal velocity $v_{n}=\mathbf{n} \cdot \partial u / \partial t$ ( $\mathbf{n}$ is the unit normal of $S$ ) satisfying the governing equation of motion given by the Mullins law $v_{n}=M \gamma \mathcal{K}$ with $M$ being a constant mobility, and $\mathcal{K}$ being the corresponding surface mean curvature. The constant $\gamma$ is taken to be the interfacial energy density (or surface tension) of all surfaces so that the total interfacial energy $E$ is given by $\gamma$ times the interfacial area. Then, a direct computation leads to

$$
\frac{d E}{d t}=-\sum_{\{S\}} \int_{S} \frac{1}{M} v_{n}^{2} d s+\gamma \sum_{\{\Gamma\}} \int_{\Gamma} v^{(\Gamma)} \cdot \sum_{j}\left(T^{(\Gamma)} \times \mathbf{n}_{j}^{(\Gamma)}\right) d l
$$

Here $\{\Gamma\}$ is the set of all $k$-junction lines of the network $(\mathrm{k}=3,4, \ldots), v^{(\Gamma)}$ is the velocity of the junction line $\Gamma$ :

$$
v^{(\Gamma)}=\frac{\partial u}{\partial t}, \quad u=u^{\left(i_{1}\right)}=\ldots=u^{\left(i_{k}\right)} \text { along } \Gamma
$$

$T^{(\Gamma)}$ is a unit tangent vector field along $\Gamma, \mathbf{n}_{j}^{(\Gamma)}$ is the unit normal of a surface containing $\Gamma$, and the index $\mathrm{j}$ runs over all surfaces which have the common edge $\Gamma$.

In order for the system to be dissipative, that is, $d E / d t \leq 0$, it is sufficient to have

$$
v^{(\Gamma)} \cdot \sum_{j}\left(T^{(\Gamma)} \times \mathbf{n}_{j}^{(\Gamma)}\right) \leq 0 .
$$

The above inequality, in fact, takes an equal sign for a triple junction when the Herring condition

$$
\sum_{j=1}^{3}\left(T \times \mathbf{n}_{j}\right)=0
$$

or equivalently

$$
\sum_{j=1}^{3} \mathbf{n}_{j}=0
$$

is enforced along a triple line $\Gamma$. That is to say, three interfaces meet along the triple junction with the angle $2 \pi / 3$. For more discussion on the Herring condition, we refer to $[1,7,13]$ and $[15]$. On the other hand, other conditions for junction lines may also be postulated to ensure that the system is dissipative. One possible example is to enforce:

$$
\sum_{j} \mathbf{n}_{j}=\lambda v^{(\Gamma)}
$$

with some relaxation parameter $\lambda \geq 0$ which may be inversely associated with a junction mobility. In this case, $\sum_{j} \mathbf{n}_{j}$ is aligned along the same direction as $v^{(\Gamma)}$. Note that since $\left(\sum_{j} \mathbf{n}_{j}\right) \cdot T^{(\Gamma)}=0$, we consequently see that for this example, the junction 
line $\Gamma$ would move in the direction perpendicular with its tangent direction. One may also consider another example given by

$$
\sum_{i} \mathbf{n}^{(i)}=\lambda T^{(\Gamma)} \times v^{(\Gamma)}
$$

with a positive constant $\lambda \geq 0$; the energy dissipation again holds in this case. We note that in our formula (4.2), there is no restriction on the exterior angles $\alpha_{i}$. Thus, the formula (4.2) is compatible with any of the above discussed conditions that enforce some conditions on the angles $\alpha_{i}$ while maintaining the energy dissipation. As a matter of fact, the generality of the angles $\left\{\alpha_{i}\right\}$ makes formula (4.2) equally applicable to junction lines with possibly negative line tensions $[15,16]$. Another applicable situation is studied in [5], where it is argued that triple junctions possess their own mobilities, and the angles along triple junctions depend on such mobilities. Triple junctions can have a low mobility, especially at a relatively low temperature, and thus the angle along a triple junction can markably deviate from the standard natural value.

In addition, while the main result of this work is an extension of the MacPhersonSrolovitz formula for three dimensional grain growth, we note that the observation made on (4.1) may be of other general interests. From (4.1), we directly obtain the formula of the mean width of a polyhedron with flat faces as discussed earlier. We may also use this formula to compute the mean width of some other common shapes in three dimensional space. For any two-dimensional domain $\mathrm{D}$, using the same idea in [9] by considering a three dimensional prism with a cross-section $\mathrm{D}$, we can deduce a generalized two dimensional von Neumann relation corresponding to arbitrary junction points as a special case:

$$
\int_{\partial D} \kappa d l+\sum_{i=1}^{n} \alpha_{i}[\partial D]=2 \pi \chi(D)
$$

which is the formula (3.1) given earlier.

Finally, to put our work in perspective, while the finding in this paper demonstrates that the equilibrium Herring conditions can be relaxed, as stipulated in [8], many other complications such as inhomogeneities and anisotropies are important for the study of grain growth in real systems. In this regard, formula (4.1) may well be useful when applying the mean width concept to a more general situation with an anisotropic energy. Meanwhile, it may also be interesting to study the stochastic variations [2]. Yet, the generalizations of (1.4) in such cases still remain elusive to us at the moment.

Acknowledgment. We would like to thank Dr. David Kinderlehrer for bringing [9] to our attention. We also like to thank him, and Drs. L.-Q. Chen, C. Liu, M. Emelianenko, A. Rollet, and P. Yu for interesting discussions on grain growth over the years. In addition, we thank the referees for their careful reading and valuable comments. We also thank Dr. M. Glicksman for alerting us the work [3] and the additional references on the subject in [4] during the final revision of our work.

\section{REFERENCES}

[1] B. Adams, S. Taäsan, D. Kinderlehrer, I. Livshits, D. Mason, C.T. Wu, W. Mullins, G. Rohrer, A. Rollett and D. Saylor, Extracting grain boundary and surface energy from measurement of triple junction geometry, Interface Science, 7, 321-337, 1999. 
[2] K. Barmak, M. Emelianenko, D. Golovaty, D. Kinderlehrer and S. Ta'asan., A new perspective on texture evolution, Intl. J. of Num. Anal. and Modeling, 5, 93-108, 2008.

[3] J.W. Cahn, The significance of average mean curvature and its determination by quantitative metallography, Trans. Metall. Soc. of AIME, 239, 610-616, 1967.

[4] M. Glicksman, P. Rios and D. Lewis, Mean width and caliper characteristics of network polyhedra, Philosophical Magazine, 89, 389-403, 2009.

[5] G. Gottstein, D.A Molodov and L.S Shvindlerman, Kinematics, dynamics, and microstructual effects of grain boundary junctions, J. Mater. Sci., 41, 7730-7740, 2006.

[6] H. Hadwiger, Vorlesungen über Inhalt, Oberfläche und Isoperimetrie, Springer, Berlin, 1957.

[7] D. Kinderlehrer and C. Liu, Evolution of grain boundaries, Math. Models Meth. Appl. Math., 4, 713-729, 2001.

[8] D. Kinderlehrer, Mathematical physics: added dimensions to grain growth, Nature, 446, 995996, 2007.

[9] R.D. MacPherson and D.J. Srolovitz, The von Neumann relation generalized to coarsening of three-dimensional microstructures, Nature, Supplementary Material, 446, 1053-1055, 2007.

[10] A. Morawiec, On equilibrium conditions at junctions of anisotropic interfaces, J. Materials Sci., 40, 2803-2806, 2005.

[11] W.W. Mullins, Two dimensional motion of idealized grain boundaries, J. Appl. Phys., 27, 900, 1956.

[12] J. Von Neumann, Written discussion of grain shapes and other metallurgical applications of topology, Metal Interfaces, Am. Soc. Metals, Cleveland, 108-110, 1952.

[13] D. Raabe, F. Roters, F. Barlat and L.Q. Chen, Continuum Scale Simulations of Engineering Materials: Fundamentals-Microstructures-Process Applications, Wiley-VCH Verlag, 2004.

[14] L. Santaló and K. Mac, Integral Geometry and Geometric Probability, Cambridge Univ. Press, 2004.

[15] J. Taylor, Mathematical models of triple junctions, Interface Science, 7, 243-249, 1999.

[16] J. Taylor, J.W. Cahn and C. Handwerker, Geometric models of crystal growth, Acta Metall. Mater., 40, 1443-1474, 1992. 